\title{
Effect of macromolecular mass transport in microgravity protein crystallization
}

\begin{abstract}
Arayik Martirosyan ${ }^{1}$, Lawrence J. DeLucas ${ }^{2}$, Christina Schmidt ${ }^{1}$, Markus Perbandt ${ }^{1}$, Deborah McCombs ${ }^{3}$, Martin Cox ${ }^{2}$, Christopher Radka ${ }^{4}$, Christian Betzel ${ }^{1}$

${ }^{1}$ Laboratory for Structural Biology of Infection and Inflammation, University of Hamburg, Hamburg, Germany; ${ }^{2}$ The Aerospace Corporation, Huntsville, AL; ${ }^{3}$ University of Alabama at Birmingham, Birmingham, AL, USA; ${ }^{4}$ St. Jude Children's Research Hospital, Memphis, TN (ORCID

Abstract 0000-0002-6037-7710)

To investigate the effect of macromolecular transport and the incorporation of protein aggregate impurities in growing crystals, experiments were performed on the International Space Station (ISS) and compared with control experiments performed in a $1 \mathrm{G}$ laboratory environment. Crystal growth experiments for hen egg-white lysozyme (HEWL) and Plasmodium falciparum glutathione S-transferase (PfGST) were monitored using the ISS Light Microscopy Module (LMM). Experiments were performed applying the liquid-liquid counter diffusion crystallization method using rectangular, optically transparent capillaries. To analyze the quantity of impurity incorporated into growing crystals, stable fluorescently labeled protein aggregates were prepared and subsequently added at different percent concentrations to nonlabeled monomeric protein suspensions. For HEWL, a covalent cross-linked HEWL dimer was fluorescently labeled, and for PfGST, a stable tetramer was prepared. Crystallization solutions containing different protein aggregate ratios were prepared. The frozen samples were launched on 19.02 .2017 via SpaceX -10 mission and immediately transferred to a $-80^{\circ} \mathrm{C}$ freezer on the ISS. Two series of crystallization experiments were performed on ISS, one during 26.02.2017 to 10.03.2017 and a second during 16.06.2017 to 23.06.2017. A comparison of crystal growth rate and size showed different calculated average growth rates as well as different dimensions for crystals growing in different positions along the capillary. The effect of macromolecular mass transport on crystal growth in microgravity was experimentally calculated. In parallel, the percentage of incorporated fluorescent aggregate into the crystals was monitored utilizing the fluorescent LMM and ground-based fluorescent microscopes.
\end{abstract}

Keywords

Light Microscopy Module $($ LMM) $・$ microgravity $\bullet$ crystal growth $\bullet$ mass transport $\bullet$ impurity incorporation

\section{Introduction}

Protein crystallography is the predominate technique used to determine the three-dimensional structure of biological macromolecules. High-resolution three-dimensional structures provide information that is used to determine the function of proteins and other biomolecules and for drug discovery in cases where proteins play a role in different disorders or diseases. Growing high-quality crystals of these biomolecules enhances the ability to obtain X-ray data at a high resolution, thereby enabling more accurate threedimensional structure determinations. Data and information obtained from crystallographic investigations are important for macromolecular engineering to optimize biomolecules for various applications in biomedical research. Macromolecular transport in crystallization processes has been shown to directly affect crystal quality (García-Ruiz et al., 2016; McPherson et al., 1999; Vekilov, 1999). Gravity-dependent flow effects, including convection and sedimentation, affect the crystal growth processes on earth (Lee and Chernov, 2002; Otálora et al., 2001; Wilcox, 1983). Density-driven solution convection might be expected to force molecules to rapidly flow past the growing crystal, thus bringing impurities such as inorganic and organic particles and macromolecular aggregates to growing crystal surfaces (Lee and Chernov, 2002). Different concentrations at different parts of a crystal created with the flow patterns may lead to nonuniform growth conditions (DeLucas et al., 1986; Wilcox, 1983). Sedimentation is another gravity-dependent effect that significantly alters the crystal growth process on earth. Depending on the density of the growing crystals versus the solution density, crystals growing in a $1 \mathrm{G}$ environment migrate to the top, bottom, or wall of a crystallization container (Carotenuto et al., 2002; DeLucas et al., 1986). Thus, sedimentation creates crystal accumulation on the surface, bottom, or wall of a crystallization container, which may interrupt further crystal growth (DeLucas et al., 1986; García-Ruiz et al., 2001a; Wilcox, 1983). Diffusion-controlled crystal growth processes in the absence of convection (and the elimination of the sedimentation effect) may be beneficial for crystal quality. Therefore, the microgravity environment appears to be ideally suited for growing crystals with improved quality

${ }^{\dagger}$ Corresponding author: Lawrence J. DeLucas

E-mail: Lawrence.delucas@aero.org 
(Kuranova et al., 2011; McPherson et al., 1999; Snell et al., 1997). In this context, it is important to investigate the effect of crystal growth rates versus crystal quality and size.

The first reported protein crystallization experiments in microgravity, performed in 1984, described the growth of lysozyme and b-galactosidase crystals on Spacelab-1 (Littke and John, 1984). Since then in subsequent space shuttle missions, unmanned satellite missions, on the Russian space station and on the International Space Station (ISS), more than 100 different proteins have been crystallized under microgravity conditions (DeLucas et al., 1986; Krauspenhaar et al., 2002; Snell et al., 1997; Timofeev et al., 2012a; Vallazza et al., 2002). In 1989, Asano et al. performed a series of crystal growth experiments for bovine pancreatic ribonuclease $S$ under microgravity conditions using the COSIMA 2 system (Asano et al., 1992; Plaas-Link and Cornier, 1988). The microgravity-grown ribonuclease $S$ single crystals revealed improved diffraction resolution compared to ground-grown crystals of equal size (Asano et al., 1992). In another experiment, crystals of uridine phosphorylase from Shewanella oneidensis MR-1 revealed improved quality for microgravity-grown crystals compared to $1 \mathrm{~g}$ controls (Safonova et al., 2012). However, some crystallization experiments performed under microgravity conditions showed no significant improvement in crystal quality (Hilgenfeld et al., 1992; Strong et al., 1992).

Macromolecule purity is a major parameter in the crystal growth process such that removal of impurities and elimination of macromolecular heterogeneity improve the probability of growing higher quality crystals (Giege et al., 1986; McPherson et al., 1999; Vekilov and Rosenberger 1996). Crystal defects often originate from incorporation of molecular aggregates and other impurities into the lattice of a growing crystal (Adawy et al., 2015; Giege et al., 1986; McPherson et al., 1999; Vekilov and Rosenberger 1996). Hen egg-white lysozyme (HEWL) has been crystallized in the presence of HEWL dimers on earth and under microgravity conditions to investigate the effect of impurity incorporation into a growing crystal. It was observed that the HEWL crystals grown under microgravity conditions contain 4.5 times less HEWL dimers than control experiments performed on earth (Carter et al., 1999). Another experiment demonstrated that the presence of chicken egg-white lysozyme dimers in the crystallization solutions in microgravity reduced crystal size, increased mosaicity, and reduced the signal-to-noise ratio of the X-ray data (Snell et al., 2001). Yet in another investigation, no significant difference in impurity incorporation between microgravity and ground crystals was observed (Snell et al., 2001). To further explore the effect of macromolecular transport phenomena in crystallization processes, experiments on the ISS were performed. For this investigation, Plasmodium falciparum glutathione S-transferase (PfGST) (Liebau et al., 2002; Perbandt et al.,
2015) and HEWL were crystallized in the presence of varying amounts of fluorescently labeled protein aggregate.

\section{Materials and Methods}

Recombinant Expression and Purification of the Proteins

PFGST from Plasmodium falciparum was expressed and purified as previously reported (Liebau et al., 2002; Perbandt et al., 2015) with few modifications. The recombinant PfGST was expressed in E. coli BL21 cells in overnight cultures at $19^{\circ} \mathrm{C}$. The cells were harvested by centrifugation and resuspended in $1^{\prime}$ phosphate-buffered saline (PBS) buffer $(\mathrm{pH}$ 7.4). The cell suspension was sonicated, and the cell debris was removed by centrifugation at $17.000^{\prime} \mathrm{g}$ at $4^{\circ} \mathrm{C}$ for $1 \mathrm{~h}$. The recombinant PfGST was purified applying glutathione affinity chromatography; additionally, PfGST tetramer was separated by gel filtration on a Superdex HiLoad 26/600 column (GE Healthcare). Monomeric hen egg lysozyme (HEWL) was obtained by subjecting commercially obtained protein (Biomedicals LLC) to size exclusion chromatography (Yarra-2000 column). To produce stable aggregates, the purified monomeric protein was subjected to crosslinking by using glutaraldehyde as the crosslinking agent. The fused protein was then reapplied to the size exclusion column to separate dimeric lysozyme from remaining monomeric protein.

\section{Dispersity Analysis of the Protein Multimeric States}

The dispersity and particle radius distribution of PfGST dimer (30 mg mL $\left.{ }^{-1}\right)$ and tetramer $\left(10 \mathrm{mg} \mathrm{mL}^{-1}\right)$ were determined and analyzed at room temperature by dynamic light scattering (DLS) applying the DLS system SpectroLightÔ 300 (Xtal Concepts $\mathrm{GmbH}$, Hamburg, Germany). Time-resolved DLS measurements of hydrodynamic radius verified the monodispersity of PfGST dimer with $R H=3.7 \pm 0.1 \mathrm{~nm}$ and tetramer with $R H=5.7 \pm 0.1 \mathrm{~nm}$ solutions, respectively. To produce stable aggregates of HEWL, monomeric protein was subjected to crosslinking as described before and subsequently run over a size exclusion column to separate remaining monomer from dimers and higher aggregates. The purified and stable dimer was then fluorescently labeled as described in the following.

\section{Fluorescent Labeling of Protein Aggregates}

To investigate the amount of impurity incorporated into growing crystals, stable fluorescently labeled protein aggregates were prepared and subsequently added at different percent concentrations to nonlabeled monomeric protein suspensions. The PfGST tetramer and lysozyme dimer, acting as impurities in the crystallization experiments, were labeled with the fluorescent dyes Alexa Fluor ${ }^{\circledR} 488$ TFP ester and Alexa 
Fluor ${ }^{\circledR} 594$ NHS ester, respectively. The Alexa Fluor ${ }^{\circledR} 488$ TFP ester $\left(\mathrm{C}_{39} \mathrm{H}_{44} \mathrm{~F}_{4} \mathrm{~N}_{4} \mathrm{O}_{11} \mathrm{~S}_{2}, M_{\mathrm{w}}=884.9 \mathrm{Da}\right)$ and Alexa Fluor® $594 \mathrm{NHS}$ ester $\left(\mathrm{C}_{39} \mathrm{H}_{37} \mathrm{~N}_{3} \mathrm{O}_{13} \mathrm{~S}_{2}, M_{\mathrm{w}}=819.8 \mathrm{Da}\right)$ were obtained from Thermo Fisher Scientific (Life Technologies). The Alexa Fluor® 488 TFP ester $(5 \mathrm{mg})$ was dissolved in $0.5 \mathrm{~mL}$ of dimethyl sulfoxide. The reactive dye solution $(80 \mu \mathrm{L})$ was slowly added to the stirring PfGST tetramer solution (10 $\mathrm{mg} / \mathrm{ml}$ in PBS). The reaction was incubated for $1 \mathrm{~h}$ at room temperature with continuous stirring. The labeled tetramer was separated from free dye using a Sephadex® G-25 prepacked gel filtration column. The column was first equilibrated with PBS buffer and then loaded with a reaction mixture and eluted with PBS buffer solution. Labeling degree (DOL) of tetramer was calculated using following calculations:

$$
\begin{aligned}
& \text { Protein concentration }(\mathrm{M})=\frac{[\mathrm{A} 280-(\mathrm{CF} \times \mathrm{A} 494)] \times \text { dilution factor }}{\varepsilon(\text { protein })} \\
& \mathrm{DOL}=(\text { moles dye }) /(\text { mole protein })=\frac{\mathrm{A} 494 \times \text { dilution factor }}{\varepsilon(\text { dye }) \times \text { protein concentration }(\mathrm{M})}
\end{aligned}
$$

The calculated DOL was approximately 4 . The protein-dye conjugate was concentrated to $10 \mathrm{mg} / \mathrm{ml}$ final concentration using Millipore Amicon ${ }^{\circledR}$ ultra-centrifugal concentrators (Merck Millipore). For lysozyme aggregate labeling, a solution of buffered lysozyme dimer at $2 \mathrm{mg} / \mathrm{mL}$ was mixed with Alexa Fluor® 594 NHS ester at $22^{\circ} \mathrm{C}$ and incubated for $1 \mathrm{~h}$. Unbound dye was removed via spin concentration $(500 \mu \mathrm{L}$ $30 M_{w}$ cutoff spin concentrator).

\section{Capillary Protein/Precipitant Filling and Sealing Proce- dure for Crystallization}

Crystallization experiments were performed using the counter/ liquid-liquid diffusion technique in capillaries of $100 \mathrm{~mm}$ length, $3 \mathrm{~mm}$ width, and $0.3 \mathrm{~mm}$ inner diameter (VitroCom). The capillaries were filled with $37 \mu \mathrm{L}$ of precipitant and 37 $\mu \mathrm{L}$ of protein solution (doped with different percentages of fluorescently labelled protein aggregate using syringes (Hamilton)). Lysozyme was prepared in 0.1 M NaOAc ( $\mathrm{pH} 4.6)$ solution with the precipitant solution consisting of $1 \mathrm{M} \mathrm{NaCl}$ and 0.1 M NaOAc ( $\mathrm{pH} 4.6)$. A sample of PfGST dimer and tetramer was prepared in $0.1 \mathrm{M} \mathrm{NaHPO}_{4}(\mathrm{pH} \mathrm{6.7)}$ solution. The precipitant solution for PfGST consisted of $2.8 \mathrm{M} \mathrm{AmSO}_{4}$, $0.1 \mathrm{M} \mathrm{NaHPO}_{4}$ and $15 \%$ glycerol ( $\mathrm{pH} \mathrm{6.7).} \mathrm{The} \mathrm{length} \mathrm{and}$ width of all flight and ground control capillaries were measured to confirm that they were within stated limits. Capillaries were visually examined before filling to ensure that there are no visual defects, e.g. cracks and jagged ends. One capillary end was filled with Apiezon $\mathrm{N}$ cryogrease (Apiezon) using a plastic $25 \mathrm{~mL}$ syringe with a rubber or silicon tube that attached the syringe with the other end fit snugly over the capillary. Grease was extruded approximately $3-5 \mathrm{~mm}$ into the capillary end. All protein and precipitant solutions were degassed using "house" vacuum prior to use for the flight and ground control experiments. The capillary was filled with protein using a glass syringe (Hamilton) with a needle small enough to fit within the width of the capillary (i.e. $<0.3 \mathrm{~mm}$ width). This was followed by layering the precipitant solution against the protein solution and filling the capillary to approximately $3 \mathrm{~mm}$ from the other end of the capillary. After completely filling the capillary with protein and precipitant solution, the opposing end was filled with Apiezon $\mathrm{N}$ grease using a plastic $25 \mathrm{~mL}$ syringe with the same rubber/silicone tube as described previously. Fiveminute drying Double Bubble epoxy (Hardman) was prepared, and each end of the capillary was dipped into the epoxy to a depth of approximately $2-3 \mathrm{~mm}$ and the capillary inspected to determine that the ends were completely sealed. The sealed capillary was then placed on a clay mount to dry for at least $5 \mathrm{~min}$. The sealed capillary was inspected using a dissecting microscope (at approximately $15^{\prime}-25^{\prime}$ magnification). The sealed capillaries were placed into a specially constructed cassette (ZIN Technologies) that allowed capillaries lie flat in cassette channels. After eight capillaries were positioned in the cassette, the top clamp (with the six washers previously placed in the screw holes) was carefully aligned and screws fastened using a hex wrench. To insure the capillaries were not damaged, the cassette top (clamp) was removed and the capillaries are visually inspected for possible cracks. If the capillaries were not damaged, the cassette top with the six washers (previously placed in the screw holes) was carefully aligned and refastened using a hex wrench. The capillary cassette was placed into a Bitran bag and then Bubble bag followed by immediate placement of the "bagged" cassette into a walk-in $-20^{\circ} \mathrm{C} \pm 2.0^{\circ} \mathrm{C}$ freezer overnight. The capillaries were reinspected for cracks after the overnight freezing process. Following the "slow freezing" of the "bagged" cassettes in the $-20^{\circ} \mathrm{C}$ freezer, the bags were immediately placed into a $-80^{\circ} \mathrm{C} \pm 20^{\circ} \mathrm{C}$ freezer and maintained in a frozen state until on-orbit activation and observation in the Light Microscopy Module (LMM).

\section{On-orbit Experiments}

Two series of experiments were performed on ISS, one during 26.02.2017 to 10.03.2017 and a second during 16.06.2017 to 23.06.2017. The cassettes were launched aboard SpaceX $(\mathrm{SpX}-10)$ in cold stowage at $-80^{\circ} \mathrm{C}$. Upon reaching the ISS, the cassettes were transferred to ISS cold stowage $\left(-80^{\circ} \mathrm{C}\right)$ while awaiting science operations. The cassettes were removed from cold stowage, thawed, and installed onto the LMM petri plate using Velcro. The LMM petri plate was installed in the LMM base adapter and the LMM prepared for powered science operations. The initial protein crystallization experiments, LMM Biophysics-1, were performed using the ISS LMM at a temperature of $20-23^{\circ} \mathrm{C}$. Powered operations began as soon as practical after installation was completed 


\section{A}
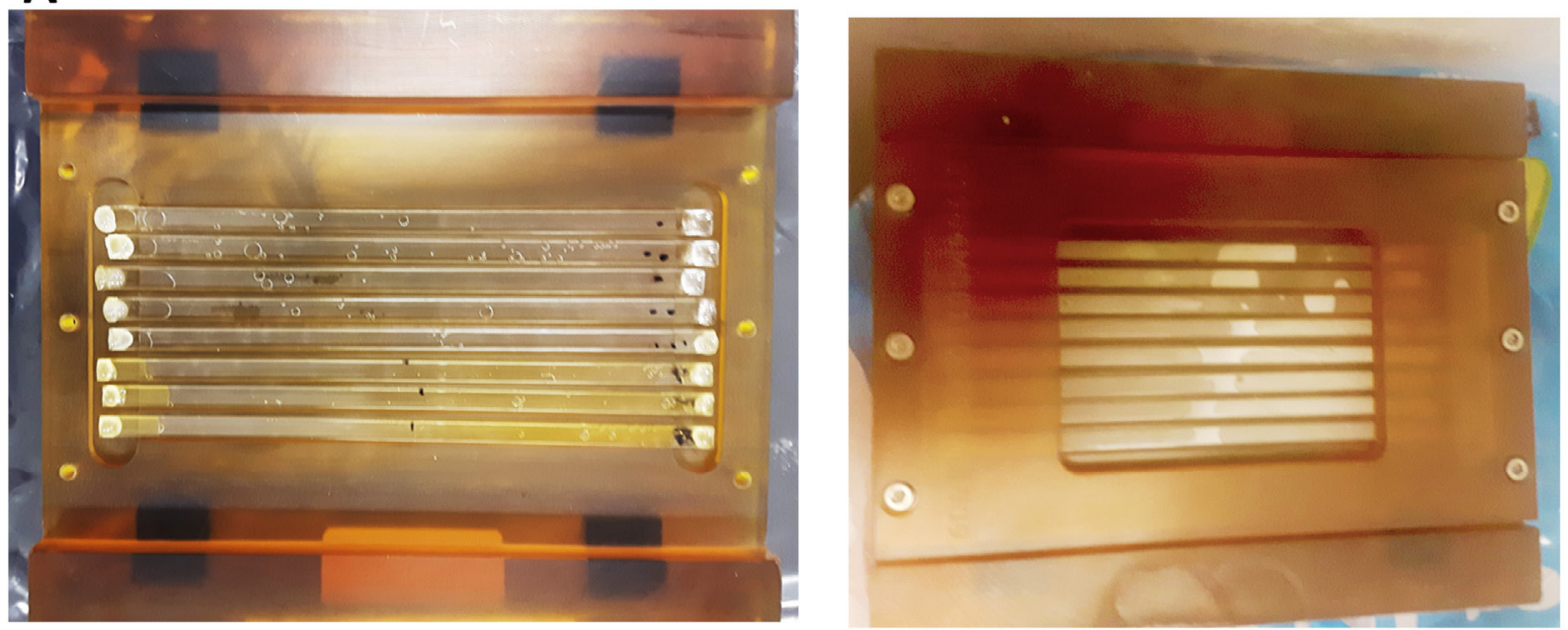

B

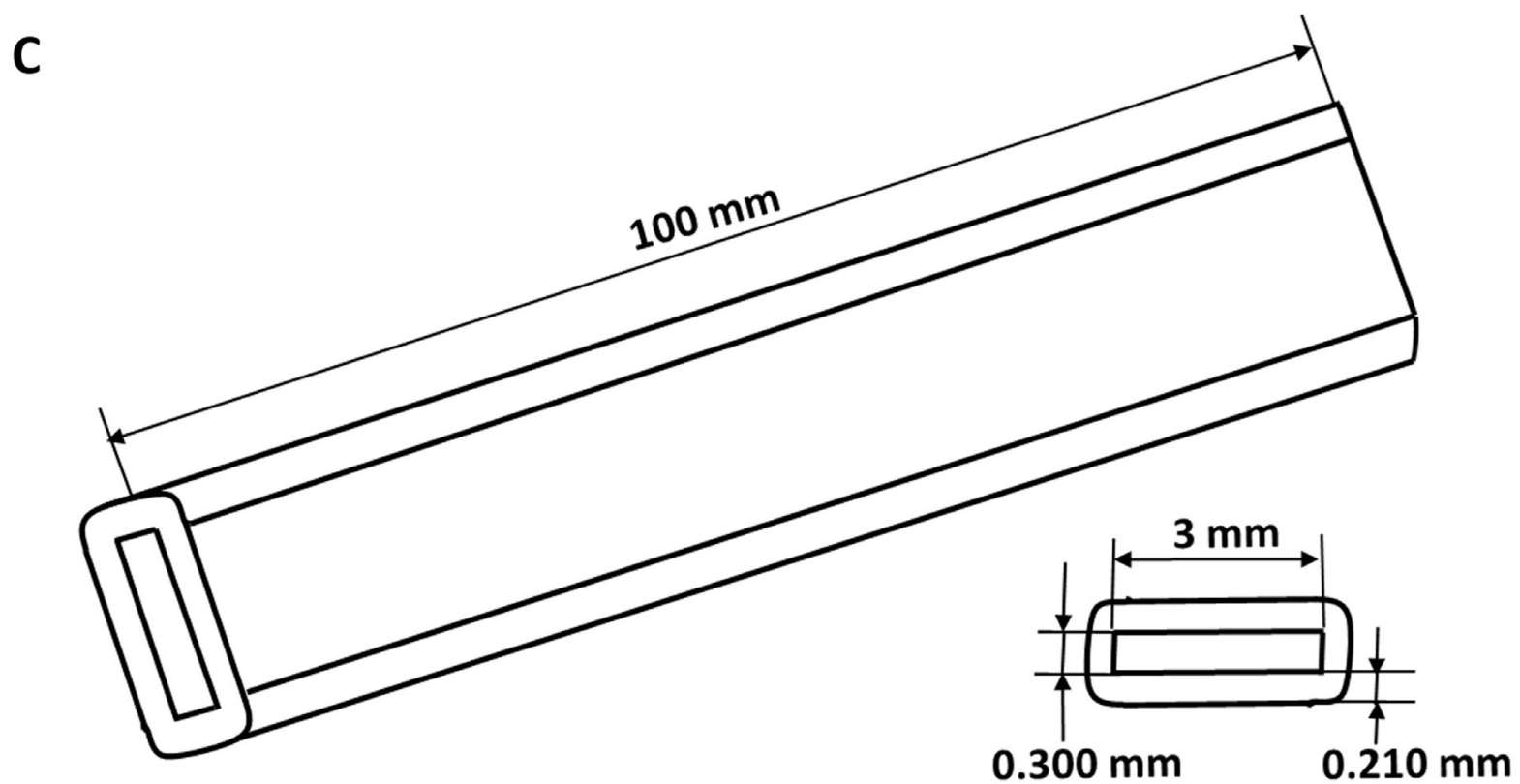

Figure 1. Hardware and capillary design for LMM Biophysics-1 protein crystallization experiments. (A) Representative images of flight cassette with eight capillaries. The left image shows a cassette with eight capillaries. The image on the right shows a cassette with aligned and fastened top cover. (B) An empty capillary. (C) Schematic diagram illustrating the capillary shape and coordinates. 
A

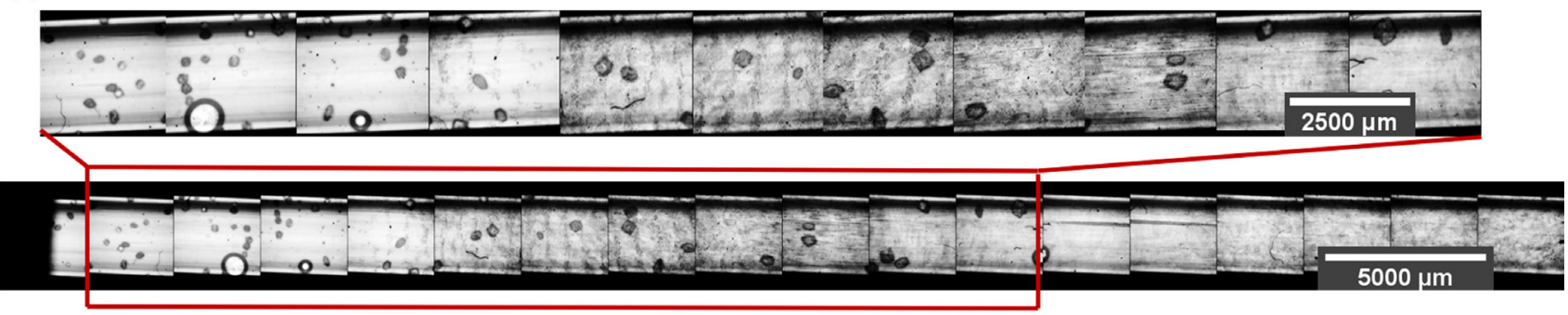

B
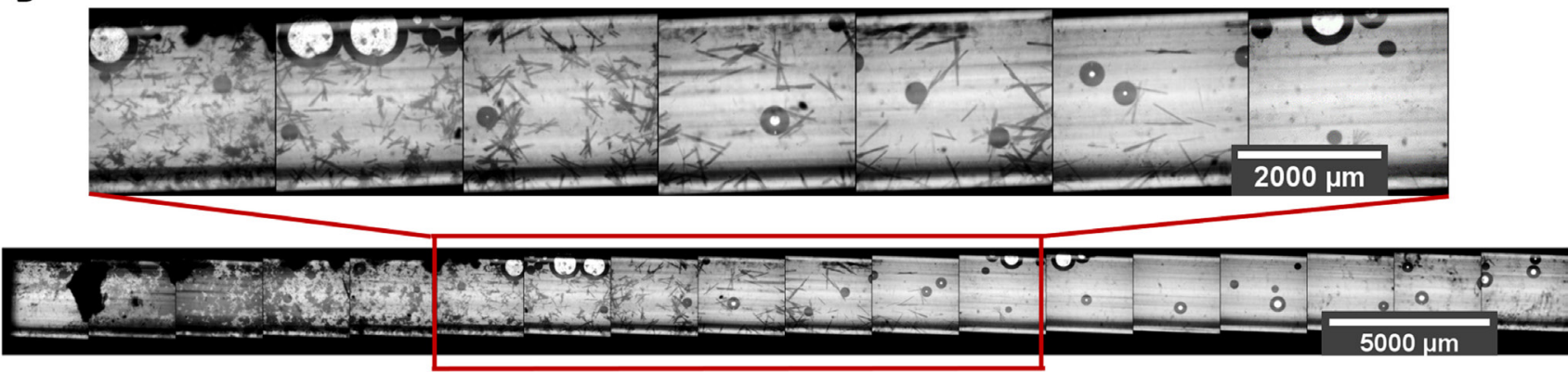

Figure 2. Protein crystallization in microgravity applying the counter diffusion technique in the capillary. Images were recorded using the ISS LMM microscope, $2.5^{\prime}$ objective. (A) Lysozyme crystals. Images were recorded at $t=8725$ min after sample thawing. (B) PfGST crystals. Images were recorded at $t=8674$ min after sample thawing.

with initiation of the crystal search phase occurring within $4 \mathrm{~h}$ of cassette installation. The growth of crystals was monitored for several days. Crystal images were recorded with 2.5', $10^{\prime}$ objectives. To investigate crystal growth rate and the fluorescence of crystals, Texas Red and FITC fluorescence filters were used.

\section{Confocal Fluorescence Imaging Experiments}

To investigate the incorporation of impurities into growing crystals for each protein, confocal fluorescence imaging experiments were performed. Crystal fluorescence images of both proteins were recorded on ISS using the LMM microscope, $2.5^{\prime}$ and $10^{\prime}$ objectives. Fluorescence imaging experiments on ground were performed using a Zeiss LSM 710 confocal laser scanning microscope (Carl Zeiss Microscopy). The capillaries were scanned with a $10^{\prime}$ magnification lens. To investigate the fluorescence of PfGST crystals, an FITC (excitation 475-495 nm; emission 515-545 nm) filter was used, and for lysozyme crystals, a Texas Red (excitation 540$580 \mathrm{~nm}$; emission 590-630 nm) filter was used.

\section{Statistical Analysis}

The image-analysis software ImageJ (developed at the National Institute of Mental Health, Maryland, USA) was then used to determine the size of crystals at different positions in the capillaries. The statistical analyses were performed using Origin (OriginLab Corporation) data analysis and graphing software tools as well as Microsoft Excel statistical tools (Microsoft Office 365 ProPlus, Microsoft Corporation). A twotailed, unpaired $t$-test (Student's $t$-test) was used to determine significant difference in mean values between different crystal groups within a $99 \%$ confidence interval.

\section{Results}

\section{Crystallization in Microgravity}

The flight cassettes were installed on the LMM microscope after sample thawing to perform crystallization experiments. Grown lysozyme crystals showed nearly similar morphologies in each capillary and were photodocumented in the LMM (Figure 2A). PfGST needle-like crystals were observed (Figure 2B). Furthermore, crystals of different sizes at different capillary areas were observed for both proteins.

The length of the major axis for lysozyme and PfGST crystals smaller in size was measured to be $150-200 \mathrm{~mm}$ and 150-320 mm, respectively, and for crystals larger in size was measured to be $450-560 \mathrm{~mm}$ and $500-780 \mathrm{~mm}$, respectively (Figure 2A and B). 


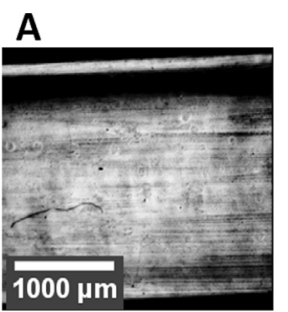

B

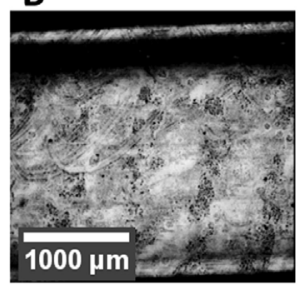

C

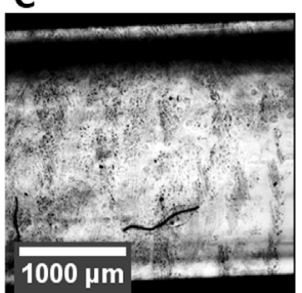

$253 \min$
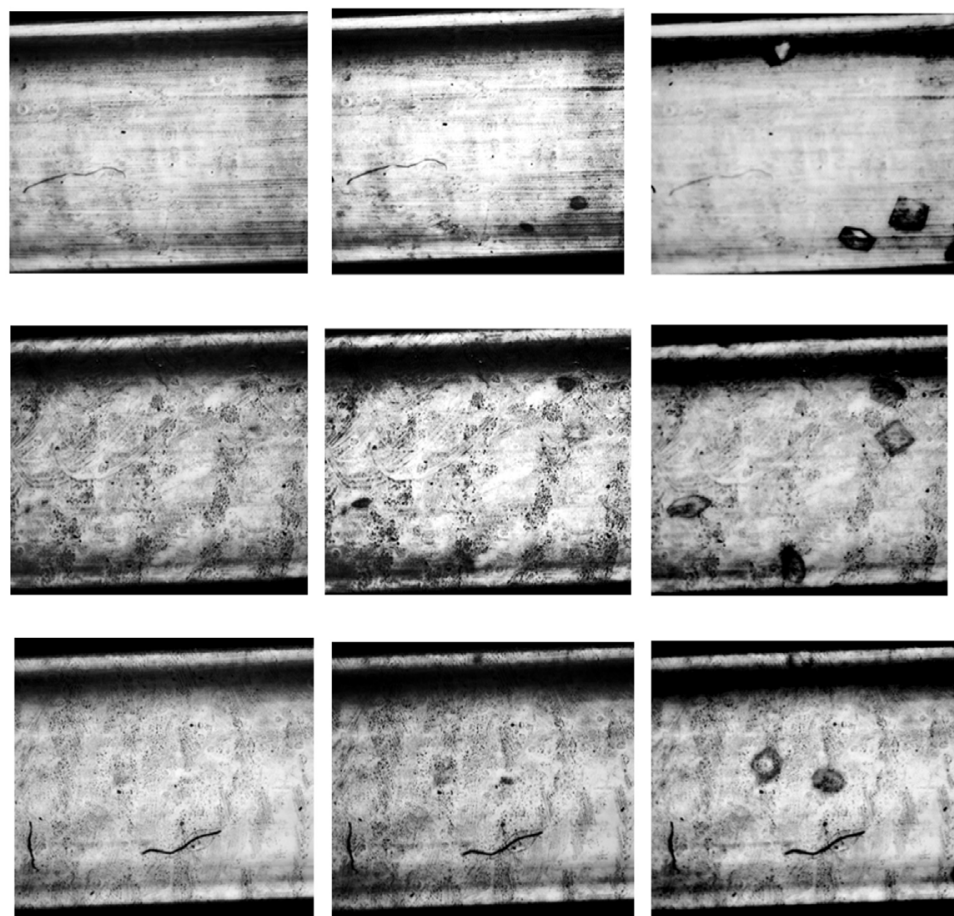

$612 \min$

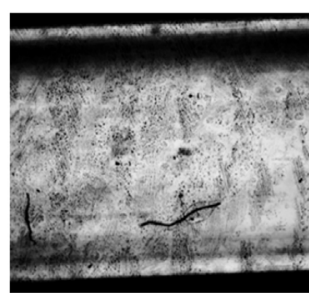

$1416 \mathrm{~min}$

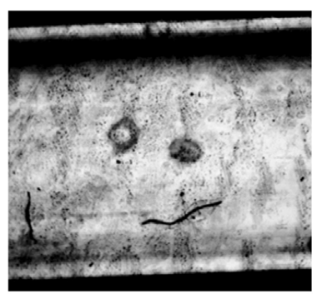

$3214 \min$
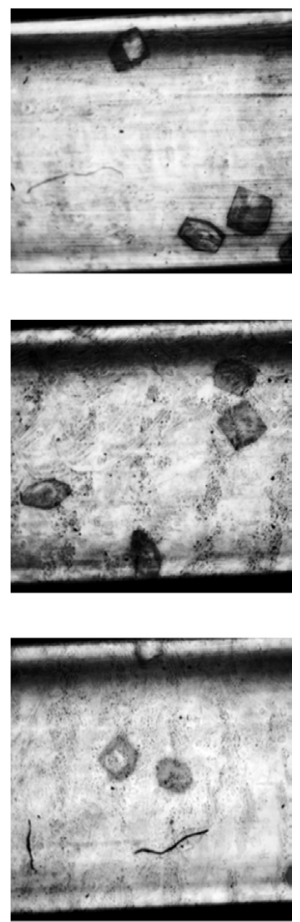

$8345 \mathrm{~min}$

Figure 3. Growth of lysozyme crystals in microgravity. Time-lapse images were recorded for three different areas (A, B, C) in capillary using LMM microscope with $2.5^{\prime}$ magnification. The imaging time extended to $t=8725$ min, first image was taken $t=253$ min after sample thawing.

\section{Crystal Growth in Microgravity}

Time-lapse images for different capillary areas were recorded to investigate crystal growth rates for lysozyme (Figure 3) and PfGST (Figure 4) crystals in microgravity. At the time points $t=253 \mathrm{~min}$ and $t=612 \mathrm{~min}$, nucleation and subsequent growth of lysozyme crystals were not observed (Figure 3). However, at time points $t=1416 \mathrm{~min}, t=3214 \mathrm{~min}$, and $t=8725 \mathrm{~min}$, growth of lysozyme crystals was observed and their growth rates were photo-documented (Figure 3).

PfGST crystals were first observed at the time point $t=3266$ min after sample thawing (Figure 4). The PfGST crystal dimensions and amount of crystals varied for several observed areas in capillary (Figure 4). The growth rate values as well as the dimensions (length of major axis) of lysozyme and PfGST crystals for different areas along the capillary were determined. The growth rate values for the $n=15$ lysozyme and $n=6$ PfGST crystals were determined.

According to the experimental results, the average growth rate $(1.9 \pm 0.23 \mathrm{~mm} / \mathrm{h})$ for lysozyme crystals with the length of major axis $<350 \mathrm{~mm}$ was significantly lower (statistical $t$-test: $p<0.01)$ than the average growth rate $(2.6 \pm 0.24 \mathrm{~mm} / \mathrm{h})$ for crystals with the length of major axis $>350 \mathrm{~mm}$ (Figure 5A). The average growth rate $(2.4 \pm 0.28 \mathrm{~mm} / \mathrm{h})$ for PfGST crystals with the length of major axis $<200 \mathrm{~mm}$ was significantly higher (Student's $t$-test: $p<0.01$ ) than the average growth rate $\left(1.2 \pm 0.13 \mathrm{~mm} / \mathrm{h}^{1}\right)$ for crystals with the length of major axis $300,400 \mathrm{~mm}$ (Figure 5B). The distribution of crystal size values (length of major axis) for lysozyme and PfGST crystals along the capillary was observed. The crystal size for both proteins at different positions in the capillaries was measured and comparatively statistically analyzed. The area in the capillary was measured from the end of capillary (i.e. the protein). Temporarily, the length of major axis values for the $n=39$ lysozyme and $n=61$ PfGST crystals was measured. The average dimensions (length of major axis) of lysozyme crystals were calculated in two areas from the capillary end, $13-25 \mathrm{~mm}$ and $25-44 \mathrm{~mm}$ with crystal sizes $277 \pm 27 \mathrm{~mm}$ and $495 \pm 47 \mathrm{~mm}$, respectively. The average size of lysozyme crystals was significantly lower (Student's $t$-test: $p<0.01$ ) for the capillary area between 13 and $25 \mathrm{~mm}$ than the average size for the capillary area between 25 and $44 \mathrm{~mm}$ (Figure 5C).

The average size of PfGST crystals was calculated in the four areas from the capillary end, $26-29 \mathrm{~mm}, 29-35 \mathrm{~mm}$, $35-41 \mathrm{~mm}$, and $41-44 \mathrm{~mm}$, with crystal sizes $158 \pm 30 \mathrm{~mm}$, $380 \pm 54 \mathrm{~mm}, 770 \pm 55 \mathrm{~mm}$, and $571 \pm 54 \mathrm{~mm}$, respectively. A significant difference for the average crystal sizes from all 

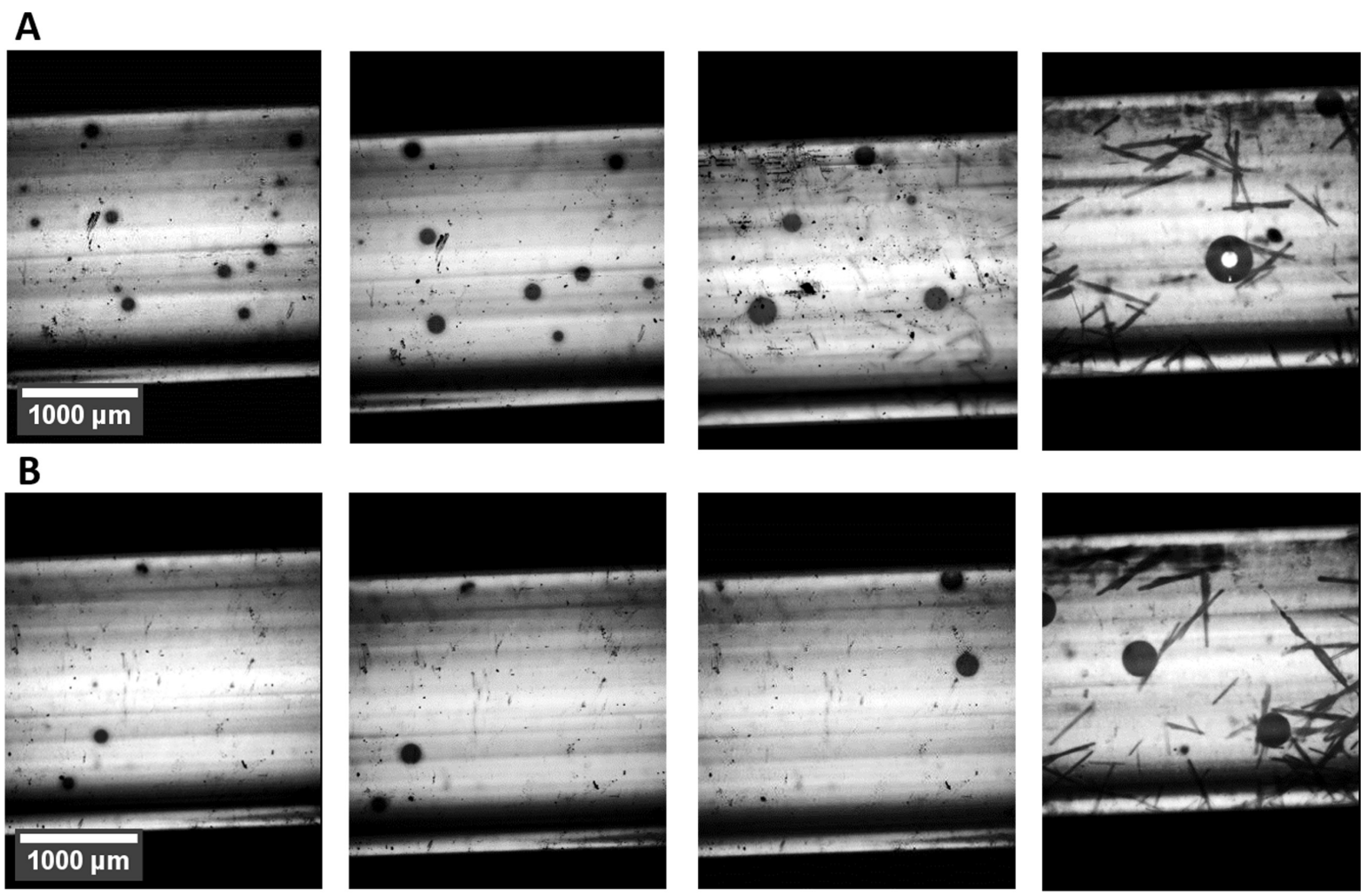

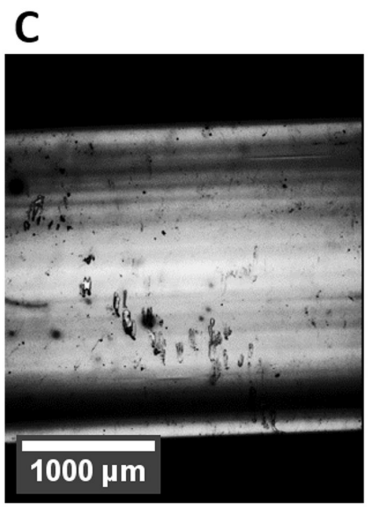

$301 \mathrm{~min}$

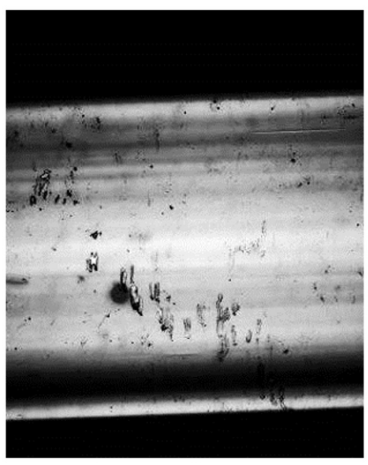

$824 \mathrm{~min}$

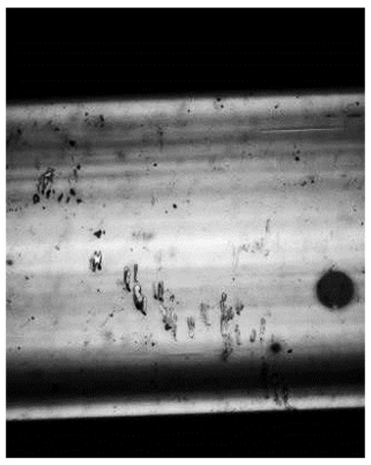

$3266 \mathrm{~min}$

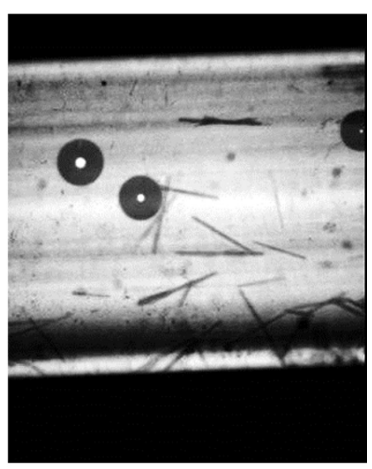

8674 min

Figure 4. Growth of PfGST crystals in microgravity. Time-lapse images were recorded for three different areas (A, B, C) in capillary using LMM microscope with $2.5^{\prime}$ magnification. The imaging time extended to $t=8674 \mathrm{~min}$, first image was taken $t=301 \mathrm{~min}$ after sample thawing.

four capillary areas (Student's t-test: $p<0.01$ ) was observed. Significance for the area $26-29 \mathrm{~mm}$ and $29-35 \mathrm{~mm}(p<0.01)$, significance for the area $26-29 \mathrm{~mm}$ and $35-41 \mathrm{~mm}(p<0.01)$, significance for the area $26-29 \mathrm{~mm}$ and $41-44 \mathrm{~mm}(p<0.01)$, significance for the area $29-35 \mathrm{~mm}$ and $35-41 \mathrm{~mm}(p<0.01)$, significance for the area 29-35 $\mathrm{mm}$ and $41-44 \mathrm{~mm}(p<0.01)$, and significance for the area $35-41 \mathrm{~mm}$ and $41-44 \mathrm{~mm}$ $(p<0.01)$ are indicated (Figure 5D).
Impurity Incorporation into Growing Crystals in Microgravity

To investigate the incorporation of impurities into growing crystals for each protein, fluorescently labeled protein aggregates were prepared that simulated protein aggregate impurities in solution. For crystallization experiments in microgravity, samples with different protein aggregate ratios were prepared. 


\section{A Growth rate for lysozyme crystals}

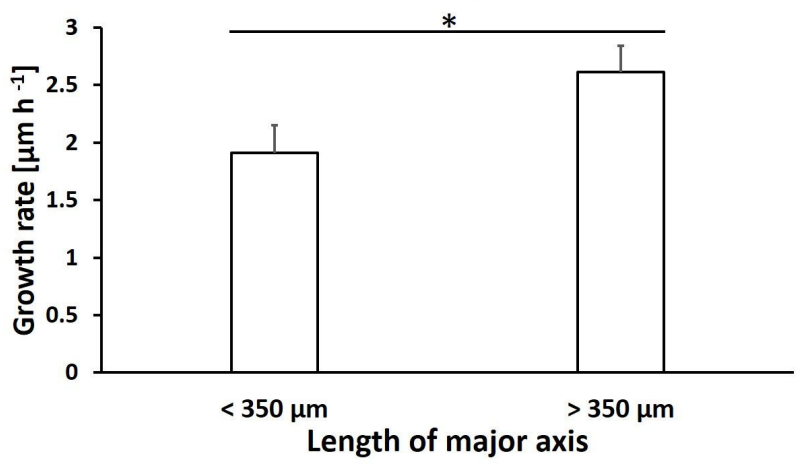

\section{Size distribution of lysozyme crystals}

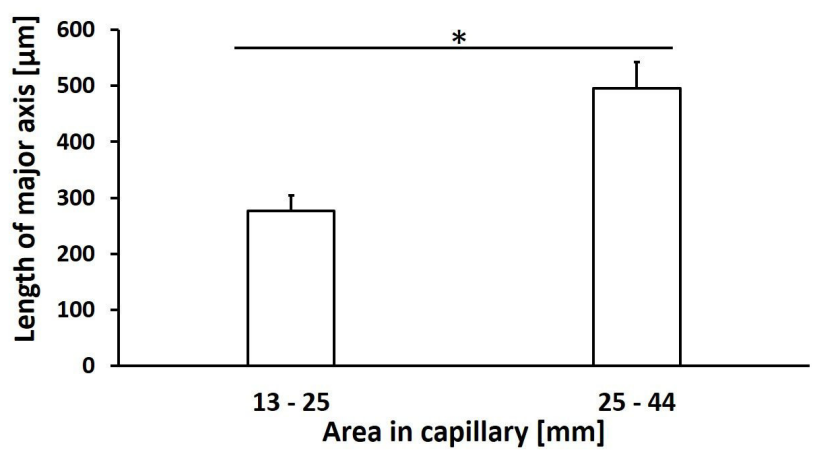

B Growth rate for PfGST crystals
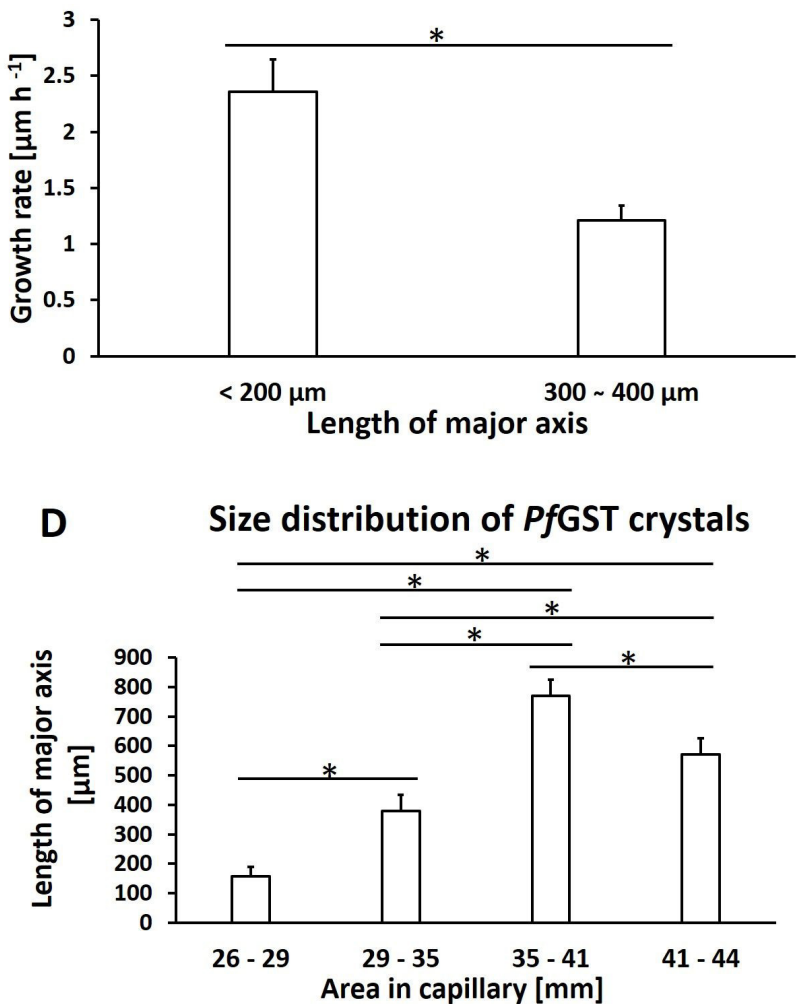

Figure 5. Comparative growth rate and size distribution values (the length of major axis) of crystals along the capillary. Different regions (areas) along the capillary were measured from the protein end of the capillary toward the protein-precipitant interface. Error bars represent standard deviations of the average. Significance within a 99\% confidence interval was determined using the Student's $t$-test and is denoted by asterisk ( $\left.{ }^{*}=p<0.01\right)$. (A) Average growth rates with standard deviations of lysozyme crystals with lengths of major axis $<350 \mathrm{~mm}$ and $>350 \mathrm{~mm}$. The sample sizes for crystals $<350 \mathrm{~mm}$ and $>350 \mathrm{~mm}$ are $n=6$ and $n=9$, respectively. (B) Average growth rates with standard deviations of PfGST crystals with lengths of major axis $<200 \mathrm{~mm}$ and $300-400 \mathrm{~mm}$. The sample sizes for crystals $<200 \mathrm{~mm}$ and $300-$ $400 \mathrm{~mm}$ are $n=3$ and $n=3$, respectively. (C) Average size distribution of lysozyme crystals for the areas from the capillary end 13-25 mm and 25-44 mm. The sample sizes for crystals in the areas 13-25 mm and 25-44 mm are $n=24$ and $n=15$, respectively. (D) Average size distribution of PfGST crystals for the areas from the capillary end $26-29 \mathrm{~mm}, 29-35 \mathrm{~mm}, 35-41 \mathrm{~mm}$, and $41-44 \mathrm{~mm}$. The sample sizes for crystals in the areas $26-29 \mathrm{~mm}, 29-35 \mathrm{~mm}, 35-41 \mathrm{~mm}$, and $41-44 \mathrm{~mm}$ are $n=15, n=20, n=18$, and $n=8$, respectively.

The LMM fluorescence micrograph of a capillary sample containing PfGST dimer and additionally fluorescently labeled PfGST aggregates (95.5:0.5 total ratio) shows the presence of fluorescence (white) in PfGST crystals grown in microgravity (Figure 6A). Initial crystallization experiments showed that fluorescently labeled PfGST aggregates are not crystallizing under same conditions as PFGST dimers. Since samples returned to the Earth, confocal images of crystals in the capillaries were recorded and photodocumented. The PfGST and lysozyme crystals grown in microgravity in the presence of fluorescence-labeled protein aggregates show the presence of fluorescence, green for PfGST crystals (Figure 6B) and red for lysozyme crystals (Figure 6C).

\section{Discussion}

Since 1984, protein crystallization experiments have been conducted in microgravity to obtain crystals of higher X-ray diffraction quality (DeLucas et al., 1986; Kundrot et al., 2001; Littke and John, 1984; McPherson et al., 1999; Snell et al., 1997). It is assumed that a convection-free, diffusioncontrolled environment will support growth of crystals of higher quality, which can be achieved using microgravity. The capillary counter diffusion method is an efficient technique to investigate diffusion-limited mass transport phenomena in macromolecular crystallization (García-Ruiz et al., 2001b; García-Ruiz, 2003). 
A
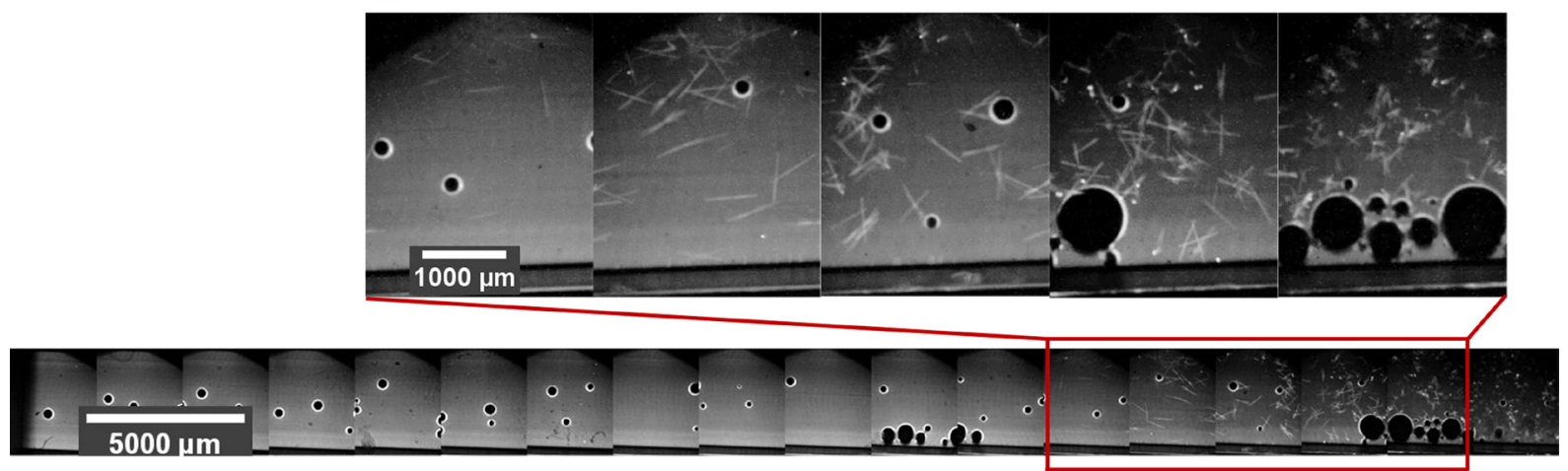

B

C
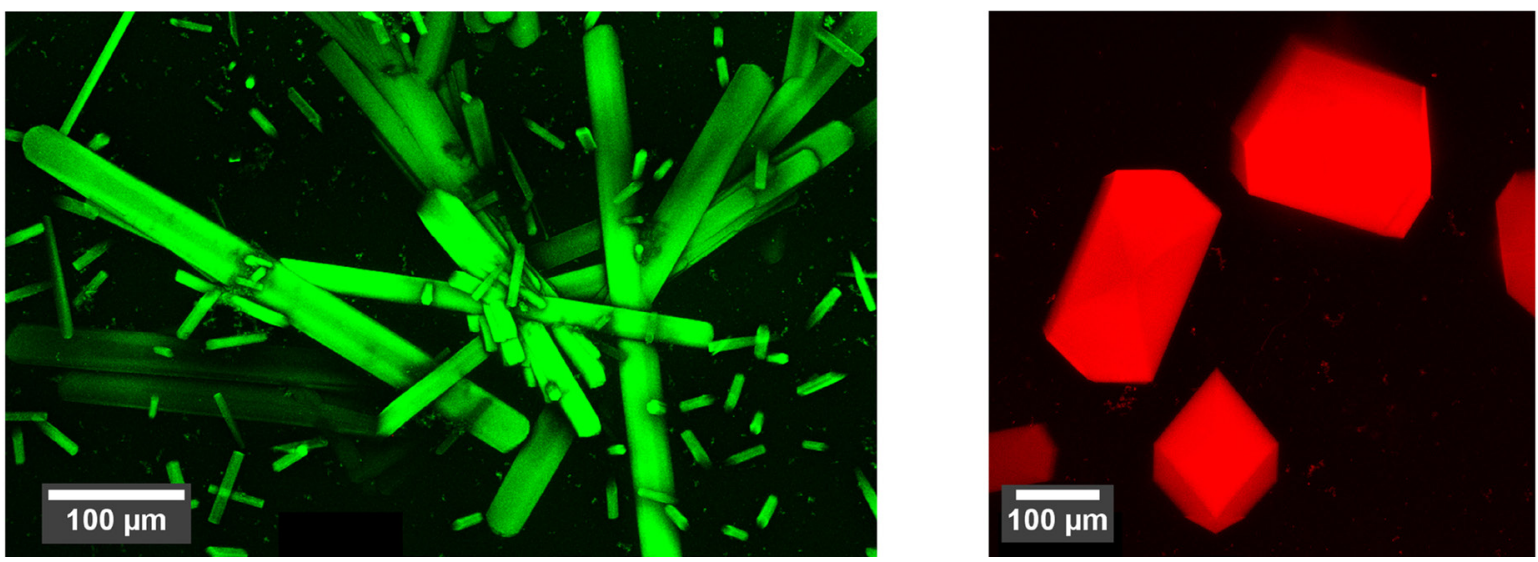

Figure 6. Crystals of PfGST and lysozyme with additionally fluorescence-labeled aggregates. (A) Crystals of PfGST dimer with additionally fluorescence-labeled (Alexa Fluor 488) PfGST aggregates. Crystal images of PfGST were recorded on ISS using the LMM microscope, 2.5' objective, with the FITC fluorescence filter. Images were recorded at $t=8742 \mathrm{~min}$ after sample thawing. (B) Crystal images of PfGST were recorded using a confocal laser microscope, 10' objective, with the FITC filter (C) Crystal images of lysozyme monomer with additionally fluorescence-labeled (Alexa Fluor 594) lysozyme aggregates were recorded using the confocal laser microscope, 10' objective, with the Texas Red filter. Images (B) and (C) were recorded after return of the samples on the earth.

This investigation addresses the following hypothesis: Improved quality of microgravity-grown protein crystals is the result of two macromolecular characteristics that exist in a buoyancy-free, diffusion-dominated solution:

- slower crystal growth rates, due to slower protein transport to the growing crystal surface and

- predilection of growing crystals to incorporate protein monomers versus higher protein aggregates due to differences in transport rates.

Experiments were performed in microgravity and in laboratory controls to compare the effect of diffusional mass transport on crystal growth rate, crystal size, and impurity incorporation. The results for both proteins, lysozyme and PfGST, reveals growth of crystals with different sizes at different positions along the capillary (Figures $2 \mathrm{~A}$ and $\mathrm{B}$ and $5 \mathrm{C}$ and $\mathrm{D}$ ). The concentration variations of the protein-precipitant solution along the length of the capillary influence the interaction of protein precipitation. In addition, the diffusive macromolecular mass transport leads to a supersaturation gradient along the capillary resulting in crystals of different size ranges along the length of the capillary (García-Ruiz, 2003; García-Ruiz et al., 2016). This is due to a gradient of crystallization conditions produced along the capillary. Changes in the average size of the lysozyme crystals were only observed in two areas within the capillary. Crystals grown between 25 and $44 \mathrm{~mm}$ along the capillary (from the capillary end) are on average significantly larger compared to crystals grown in the area between 13 and $25 \mathrm{~mm}$ (Figure $5 \mathrm{C}$ ). Analogous to the lysozyme crystallization results, changes in the average sizes of the PfGST crystals were observed such that the average sizes of PfGST crystals increased in the capillary area from 26 to $41 \mathrm{~mm}$ from the 
capillary end (also it started to decrease again in the area from 41 to $44 \mathrm{~mm}$ along the capillary; Figure 5D). As the counter diffusion method is based on mixing protein and precipitate solution by diffusion in opposite directions, it is assumed that lysozyme molecules diffuse an order of magnitude more slowly than the precipitant. This is caused by the fact that proteins reveal lower diffusion coefficient values in liquid environments compared to the diffusion coefficient values of the small molecule (salt ions) precipitant (Brune and Kim, 1993; Tyn and Gusek, 1990). Therefore, the concentration of precipitant in a capillary containing lysozyme increases at a faster rate than the concentration reduction of lysozyme in the same area of the capillary. In this context, the concentration of lysozyme in the area of the capillary initially containing the precipitant increases at a slower rate than the reduction of concentration of the precipitant in the same area of the capillary. This process produces an initial supersaturation wave in the protein area/side of the capillary, subsequently generating nucleation events and crystal growth (GarcíaRuiz et al., 2001b). This explains the observed growth of lysozyme and PfGST crystals in the initial protein area within the capillaries. It is supposed that nucleation and subsequent crystal growth occur at high protein and precipitant concentration values (at the interface between the protein and precipitant) and also at a high value of supersaturation and nucleation degree (Otálora et al., 2009). The supersaturation degree and the diffusion coefficient values change along the capillary over time, generating additional nucleation events. The supersaturation rate decreases with time, and the nucleation process and crystal growth occur at slower rates (Otálora et al., 2009; García-Ruiz et al., 2016). This effect of diffusional mass transport on nucleation and crystal growth is responsible for the observation of different crystal dimensions and different average growth rates for crystals in different positions along the capillary (Figures 2 and 5). Presumably within the diffusional gradients, crystal dimensions change proportionally to changes in nucleation and growth rates of crystals. As a result, the process whereby a gradient of precipitant concentrations naturally results in a position in the capillary with specific nucleation and growth rates that produce larger and improved crystal quality. For lysozyme, the estimated position with certain conditions optimal for the crystallization corresponded to the area $25-44 \mathrm{~mm}$ from the capillary end (Figure $5 \mathrm{C}$ ). The size distribution of PfGST crystals presented in Figure 5D shows that the on average, the largest crystals were grown in the area 35$41 \mathrm{~mm}$. It is assumed that in this area, conditions optimal for crystallization were originated over time by the diffusioncontrolled macromolecular mass transport.

In previously reported studies, crystallization experiments were performed to investigate the relation between impurity incorporation and protein crystal growth process (Adawy et al., 2015; Carter et al., 1999; Snell et al., 2001). Our study involves fluorescently labeled protein aggregates to represent the impurities in the growth solution. The PfGST protein solution contained $0.5 \%$ of fluorescently labeled PfGST aggregates (Figure 6A). The analyzed data with respect to impurity incorporation into growing crystals are displayed in Figure 6. The LMM fluorescence micrograph and the confocal microscopy images of PfGST and lysozyme crystals show the presence of fluorescence in crystals. These initial data indicate that the crystals incorporated notable quantities of fluorescently labeled impurities.

In summary, the manuscript reports that comparative crystallization experiments using the ISS LMM facility were successfully performed in microgravity using an innovative capillary counter diffusion technique. Future studies will involve a statistical analysis of the percentage incorporation of impurities (via quantitative analysis of total fluorescence/crystal volume) incorporated into microgravity versus unit gravity grown crystals. This future research will address hypothesis 2: the diffusion-limited crystal growth environment producing a self-purifying process may dominate due to slower transport rates for larger aggregates in a diffusion-limited environment. This hypothesis assumes that crystal growth in microgravity benefits from a principally purer solution (less aggregate incorporation) than crystals in the presence of convective forces (Carter et al., 1999; McPherson et al., 1999).

\section{Acknowledgments}

The authors would like to thank all the people who have been involved in the SpaceX SpX-10 mission. The authors would like to thank all the crew members of ISS expedition 50 and 52. The authors would also like to thank all the participants (i.a. Zin Technologies) of Biophysics-1 project. This research has been supported by the NASA (Grant No. 80NSSC18K0013) and Deutsche Luft und Raumfahrt Agentur (DLR) (Grant No. 50WB1423). The authors also acknowledge UAB High Resolution Imaging Service Center, Shelby 135c, Confocal/Light Microscopy Core at the University of Alabama at Birmingham.

\section{References}

Adawy A, van der Heijden EG, Hekelaar J, van Enckevort WJ, de Grip WJ, Vlieg E (2015) A comparative study of impurity effects on protein crystallization: Diffusive versus convective crystal growth. Crystal Growth \& Design 15(3), 1150-1159

Asano K, Fujita S, Senda T, Mitsui Y (1992) Crystal growth of ribonuclease $S$ under microgravity. Journal of Crystal Growth 122, 323-329 
Brune D, Kim S (1993) Predicting protein diffusion coefficients. Proceedings of the National Academy of Sciences 90(9), 3835-3839

Carotenuto L, Cartwright JHE, Castagnolo D, Ruiz JG, Otálora F (2002). Theory and simulation of buoyancy-driven convection around growing protein crystals in microgravity. Microgravity Science and Technology 13(3), 14

Carter DC, Lim K, Ho JX, Wright BS, Twigg PD, Miller TY, Chapman J, Keeling K, Ruble J, Vekilov PG, Thomas BR, Rosenberger F, Chernov AA (1999) Lower dimer impurity incorporation may result in higher perfection of HEWL crystals grown in microgravity: A case study. Journal of Crystal Growth 196, 623-637

DeLucas LJ, Suddath FL, Snyder R, Naumann R, Broom MB, Pusey M, Yost V, Herren B, Carter D, Nelson B, Meehan E J, McPherson A, Bugg CE (1986) Preliminary investigations of protein crystal growth using the space shuttle. Journal of Crystal Growth 76, 681-693

García-Ruiz JM (2003) Counterdiffusion methods for macromolecular crystallization. In Methods in Enzymology, Vol. 368. Academic Press, pp 130-154

García-Ruiz JM, Novella ML, Moreno R, Gavira JA (2001a) Agarose as crystallization media for proteins: I: Transport processes. Journal of Crystal Growth 232(1-4), 165-172

García-Ruiz JM, Otálora F, Novella M, Gavira JA, Sauter C, Vidal O (2001b) A supersaturation wave of protein crystallization. Journal of Crystal Growth 232(1-4), 149-155

García-Ruiz JM, Otálora F, García-Caballero A (2016) The role of mass transport in protein crystallization. Acta Crystallographica Section F: Structural Biology Communications 72(2), 96-104

Giege R, Dock AC, Kern D, Lorber B, Thierry JC, Moras D (1986) The role of purification in the crystallization of proteins and nucleic acids. Journal of Crystal Growth 76(3), 554-561

Hilgenfeld R, Liesum A, Storm Rd, Plaas-Link A (1992) Crystallization of two bacterial enzymes on an unmanned space mission. Journal of Crystal Growth 122, 330-336

Krauspenhaar R, Rypniewski W, Kalkura N, Moore K, DeLucas L, Stoeva S, Mikhailov A, Voelter W, Betzel C (2002) Crystallisation under microgravity of mistletoe lectin I from Viscum album with adenine monophosphate and the crystal structure at $1.9 \mathrm{~A}$ resolution. Acta Crystallographica Section D: Biological Crystallography 58, 1704-1707

Kundrot CE, Judge RA, Pusey ML, Snell EH (2001) Microgravity and macromolecular crystallography. Crystal Growth \& Design 1(1), 87-99

Kuranova IP, Smirnova EA, Abramchik YA, Chupova LA, Esipov RS, Akparov VK, Kovalchuk MV (2011) Crystal growth of phosphopantetheine adenylyltransferase, carboxypeptidase $t$, and thymidine phosphorylase on the international space station by the capillary counter-diffusion method. Crystallography Reports. 56(5), 884

Lee CP, Chernov AA (2002) Solutal convection around growing protein crystals and diffusional purification in space. Journal of Crystal Growth 240(3), 531-544

Liebau E, Bergmann B, Campbell AM, Teesdale-Spittle P, Brophy PM, Lüersen K, Walter RD (2002) The glutathione S-transferase from
Plasmodium falciparum. Molecular and Biochemical Parasitology 124(1), 85-90

Littke W, John C (1984) Protein single crystal growth under microgravity. Materials 225, 203-204

McPherson A, Malkin AJ, Kuznetsov YG, Koszelak S, Wells M, Jenkins G, Howard J, Lawson G (1999) The effects of microgravity on protein crystallization: Evidence for concentration gradients around growing crystals. Journal of Crystal Growth 196, 572-586

Otálora F, Gavira JA, Ng JD, García-Ruiz JM (2009) Counterdiffusion methods applied to protein crystallization. Progress in Biophysics and Molecular Biology 101(1-3), 26-37

Otálora F, Novella ML, Gavira JA, Thomas BR, García-Ruiz JM (2001) Experimental evidence for the stability of the depletion zone around a growing protein crystal under microgravity. Acta Crystallographica Section D: Biological Crystallography 57(3), 412-417

Perbandt M, Eberle R, Fischer-Riepe L, Cang H, Liebau E, Betzel C (2015) High resolution structures of Plasmodium falciparum GST complexes provide novel insights into the dimer-tetramer transition and a novel ligand-binding site. Journal of Structural Biology 191(3), 365-375

Plaas-Link A, Cornier J (1988) Concepts for crystallization of organic material under microgravity. Applied Microgravity Technology 3, 123

Safonova TN, Mordkovich NN, Polyakov KM, Manuvera VA, Veiko VP, Popov VO (2012) Crystallization of uridine phosphorylase from Shewanella oneidensis MR-1 in the laboratory and under microgravity and preliminary X-ray diffraction analysis. Acta Crystallographica Section F: Structural Biology and Crystallization Communications 68, 1387-1389

Snell EH, Cassetta A, Helliwell JR, Boggon TJ, Chayen NE, Weckert E, Holzer K, Schroer K, Gordon EJ, Zagalsky PF (1997) Partial improvement of crystal quality for microgravity-grown apocrustacyanin C1. Acta Crystallographica Section D 53(3), 231-239

Snell EH, Judge RA, Crawford L, Forsythe EL, Pusey ML, Sportiello M, Todd P, Bellamy H, Lovelace J, Cassanto JM (2001) Investigating the effect of impurities on macromolecule crystal growth in microgravity. Crystal Growth \& Design 1, 151-158

Strong RK, Stoddard BL, Arrott A, Farber GK (1992) Long duration growth of protein crystals in microgravity aboard the MIR space station. Journal of Crystal Growth 119, 200-214

Timofeev V, Smirnova E, Chupova L, Esipov R, Kuranova I (2012a) Xray study of the conformational changes in the molecule of phosphopantetheine adenylyltransferase from Mycobacterium tuberculosis during the catalyzed reaction. Acta Crystallographica Section D: Biological Crystallography 68(12), 1660-1670

Tyn MT, Gusek TW (1990) Prediction of diffusion coefficients of proteins. Biotechnology and Bioengineering 35(4), 327-338

Vallazza M, Banumathi S, Perbandt M, Moore K, DeLucas L, Betzel C, Erdmann VA (2002) Crystallization and structure analysis of Thermus flavus 5S rRNA helix B. Acta Crystallographica Section D: Biological Crystallography 58(10), 1700-1703 
Vekilov PG, Rosenberger F (1996) Dependence of lysozyme growth kinetics on step sources and impurities. Journal of Crystal Growth 158(4), 540-551

Vekilov PG (1999) Protein crystal growth-Microgravity aspects. Advances in Space Research 24, 1231-1240

Wilcox WR (1983) Influence of convection on the growth of crystals from solution. Journal of Crystal Growth 65(1-3), 133-142 